\section{EXPERT
REVIEWS}

\title{
Scientific advances in headache research: an update on neurostimulation
}

Expert Rev. Neurother. 13(1), 15-17 (2013)

\section{Jan Hoffmann*1 and Delphine Magis ${ }^{2}$}

'Department of Neurology, Charité - Universitätsmedizin Berlin, Charitéplatz 1, 10117 Berlin, Germany ${ }^{2}$ Headache Research Unit, Department of Neurology, University of Liège, Boulevard du 12ème de Ligne 1, 4000 Liège, Belgium *Author for correspondence: Tel.: +4930450560276 Fax: +4930450560912 jan.hoffmann@charite.de

\section{3rd European Headache and Migraine Trust International Congress London, UK, 20-23 September 2012}

The pathophysiological understanding of migraine and other primary headaches has been substantially improved over the last 20 years. A milestone that paved the way for successful research was the development of the International Classification of Headache Disorders published by the International Headache Society in 1988. The classification facilitated a clear clinical diagnosis of headache disorders and allowed research efforts to be focused on clearly defined syndromes. Recent advances in the understanding of headache disorders have been driven by the availability of new research tools, such as advanced imaging techniques, genetic tools, pharmaceutical compounds and devices for electrical or magnetic stimulation. The latest scientific and clinical advances were presented at the recent European Headache and Migraine Trust International Congress (EHMTIC) in London (UK).

The European Headache and Migraine Trust International Congress (EHMTIC) was held from 20 to 23 September in London, UK. The scientific program of this biennial congress included several informative teaching courses on experimental techniques for migraine research as well as clinical management of migraine and other primary headaches. Tobias Kurth from Bordeaux University (Bordeaux, France) opened the congress with the MacDonald Critchley lecture on migraine genetics epidemiology. The scientific core of the congress were multiple poster sessions covering basic laboratory and experimental clinical science on novel aspects of the pathophysiology of headache disorders, clinical aspects of headache care and recent advances in the acute and preventative treatment of migraine. Beside the poster sessions, lectures were given for the awarding of the Enrico Greppi Award and the Giuseppe Nappi Cluster Headache Award conferred to Sait Ashina (Beth Israel Medical Center, NY, USA) and Delphine Magis (University of Liège, Liège, Belgium) for their respective research contributions on depression and risk of transformation of episodic to chronic migraine, and central mechanisms of occipital nerve stimulation (ONS) in cluster headache. The Migraine Trust lecture was given by Karl
Messlinger (University of Erlangen-Nürnberg, Germany), who provided an extensive overview of the neurobiology of migraine. Finally, in the Jes Olesen EHF lecture Rigmor Jensen (Danish Headache Center, Copenhagen, Denmark) presented the recent advances and challenges in headache research.

Neurostimulation is of increasing interest for the treatment of primary headaches. Initial experiences with invasive methods, such as deep brain stimulation for drug-refractory cluster headache, have been effective but were hampered by severe side effects. Research efforts are now being focused on noninvasive or minimally invasive neurostimulation therapies that could be used in less disabled patients. A variety of neurostimulation devices and first data about their potential efficacy were presented at the conference and are reviewed here.

\section{Noninvasive neurostimulation devices Transcranial magnetic stimulation}

Transcranial magnetic stimulation (TMS) is an established method to modulate cortical excitability and has been used for several neurological purposes for many years. Holland et al. demonstrated in an in vivo model of migraine, that single-pulse TMS (sTMS) was able to inhibit 
cortical spreading depression [1]. A recent randomized controlled trial (RCT) suggested a positive, although modest effect of sTMS for the acute treatment of migraine with aura [2]. At this year's EHMTIC, sTMS was presented in the context of a postmarket pilot program of the SpringTMSTM device for the acute treatment of migraine [3]. In this study, 37 patients (24 migraine with aura, and 13 migraine without aura) used the device in an outpatient setting over three consecutive months. A total of 504 attacks of migraine with aura and 273 attacks of migraine without aura were treated. A significant reduction or alleviation of migraine pain was reported by $73 \%$ of the patients, while an improvement of migraine-associated symptoms was reported by $63 \%$. No adverse events were reported. Taken together with the previous sTMS RCT, these results indicate that sTMS could be a promising acute migraine treatment. However the sample size was small and objective data from headache diaries are lacking. Further well-conducted RCTs and pharmacoeconomic data are warranted. In his invited new scientist lecture, A Vigano (Sapienza University of Rome, Rome, Italy) explained that excitatory intermittent theta burst repetitive pulse TMS (rTMS) of the visual cortex (i-TBS) applied to healthy volunteers could induce changes in cortical responsiveness, which should be able to normalize the cortical interictal neurophysiological abnormalities found in migraineurs, and so become a valuable migraine preventive treatment [4]. Clinical results of i-TBS in episodic migraineurs are eagerly awaited.

\section{Transcranial direct current stimulation}

Besides TMS, transcranial direct current stimulation (tDCS) is another central neuromodulatory technique that is able to modify neuronal firing, but previous trials performed in migraine disclosed inconsistent results [5]. Vigano presented the preliminary results of a proof-of-concept trial of excitatory anodal tDCS of the visual cortex performed in seven episodic migraineurs as preventive treatment [6]. After 2 months of treatment with anodal tDCS, there was on average a significant reduction in migraine frequency $(-36.65 \%)$ and attack duration $(-43.25 \%)$, and the interictal habituation of visual evoked potentials tended to increase. Larger RCTs with anodal tDCS may thus be worthwhile in migraine.

\section{Transcutaneous vagus nerve stimulation}

Magis and Jean Schoenen (University of Liège) presented data about their initial experience with a transcutaneous vagus nerve stimulation (tVNS) device (Gammacore ${ }^{\circledR}$ ). Twelve migraine without aura (five patients with medication-overuse headache), four trigeminal autonomic cephalalgia (TAC) and two hemicrania continua (HC) patients were included. Neurostimulation was performed three times daily over $90 \mathrm{~s}$ as preventive therapy. At the time of presentation, results were available for 13 patients. Out of these, ten patients had stopped the treatment before completion of the study due to lack of efficacy and/or significant side effects. A total of three patients (one medication overuse headache, one TAC and one $\mathrm{HC}$ ) experienced a significant reduction in attack frequency, in two of them the benefit remained after 7-month follow-up [7]. In another trial, Nesbitt et al. used tVNS to treat 14 patients suffering from cluster headache $(\mathrm{CH}$; seven chronic $\mathrm{CH}$ s and seven episodic $\mathrm{CH}$ ) during a median period of 13 weeks. Mean subjective improvement was $60 \%$ in 13 patients. Twelve patients were able to reduce or even stop their previous preventive treatment. The same authors also used tVNS to relieve pain in two HC patients previously treated with ONS [8]. Taken together, these results indicate that more trials are needed to clarify a potential efficacy of $\mathrm{tVNS}$ and that treatment of TACs could be a valuable indication.

\section{Transcutaneous supraorbital stimulation}

Case reports using invasive supraorbital stimulation have shown promising results [5]. Along the same line, the efficacy and safety of a transcutaneous supraorbital nerve stimulator (tSNS), the Cefaly ${ }^{\circledR}$ device, has been tested in a multicenter, double-blind, randomized, sham-controlled trial in 67 episodic migraineurs [9]. Patients were randomly assigned to verum or sham stimulation after a 1-month run-in period. tSNS was performed daily for 20 min during 3 months. The authors observed a significant reduction of monthly migraine days and attacks (both $\mathrm{p}=0.04$ ) and acute antimigraine drug intake $(p=0.007)$. The $50 \%$ responder rate was significantly higher in the verum $(38.1 \%)$ than in the sham group (12.1\%). There were no adverse events. These results suggest that $\mathrm{tSNS}$ with the Cefaly device might offer a promising alternative to drugs for the preventive treatment of migraine. Hence, if its effectiveness is slightly lower than the best pharmacologic prophylactic treatment, such as topiramate, the absence of adverse events constitutes a real advantage.

\section{Invasive neurostimulation devices}

\section{Sphenopalatine ganglion neurostimulation}

Schoenen presented the final results of the multicenter European Pathway CH-1 RCT on the efficacy of sphenopalatine ganglion stimulation (SPGS) for the treatment of chronic $\mathrm{CH}$, using the ATI $^{\circledR}$ implantable microstimulator [10]. The preliminary results reported at the annual meeting of the American Academy of Neurology indicated that SPGS induced a significant reduction of frequency and intensity of CH [11]. The SPGS ATI device is surgically implanted into the pterygopalatine fossa and it electrically stimulates the sphenopalatine ganglion to disrupt the trigeminal parasympathetic reflex that involves the sphenopalatine ganglion. Stimulation is triggered by the patient using a handheld remote control. Available data suggest that this microstimulator is MR-safe. The blinded experimental period of the $\mathrm{CH}-1$ trial was completed by 27 patients. Pain relief was achieved in $67 \%$ of headache attacks with full stimulation versus $8 \%$ for placebo. Twenty six percent of patients experienced a significant pain relief in $\geq 50 \%$ of the attacks, $37 \%$ reported a frequency improvement of at least $50 \%$ and $7 \%$ had both effects. As a result of the surgical procedure $47 \%$ of the patients experienced transient and mild-to-moderate numbness in the second branch of the trigeminal nerve.

\section{Occipital nerve stimulation}

Several open trials have recently suggested that ONS could be effective in the prevention of drug-resistant chronic $\mathrm{CH}$ [12-16] and, to a lesser extent, chronic migraine [17-19]. 
During the conference J Vesper et al. (Heinrich-Heine University Hospital, Düsseldorf, Germany) presented a study in which they investigated the outcome of 20 patients with various therapy-refractory chronic headaches under ONS. Nine patients suffered from chronic migraine, one patient had a thalamic infarction, one $\mathrm{CH}$, four tension type headache and five recurrent cervicogenic headache. Patients were followed up over 3 months. A total of 16 out of the 20 patients experienced a significant reduction in pain intensity. No adverse events were reported. This study on a variety of refractory headache types well illustrates that the mechanism of action of ONS in headache is absolutely nonspecific and does not act on the disease generator, as suggested before [15,20].

\section{Conclusion}

The high amount of neurostimulation trials and case reports presented at the 3rd EHMTIC contrasts with the obvious lack of new specific drugs in the headache field. Hence, no acute migraine-specific drugs have been released since the advent of triptans 20 years ago, and the hopes raised by gepants (CGRP antagonists), ditans $\left(5 \mathrm{HT}_{1 \mathrm{~F}}\right.$ agonists) and the CSD-blocker tonabersat are somewhat disappointing. Central neuromodulation techniques such as TMS or tDCS could become the first migraine-specific preventive therapies, as all preventive drugs used up to now are not specific to the disease. Even if the preliminary results obtained with minimally and noninvasive neurostimulation therapies in headache are promising, large well-conducted RCTs are missing for most devices and optimal device settings and mode of action are unknown.

\section{Financial \& competing interests disclosure}

The authors have no relevant affiliations or financial involvement with any organization or entity with a financial interest in or financial conflict with the subject matter or materials discussed in the manuscript. This includes employment, consultancies, honoraria, stock ownership or options, expert testimony, grants or patents received or pending, or royalties.

No writing assistance was utilized in the production of this manuscript.

\section{References}

1 Holland PR, Schembri C, Fredrick J, Goadsby PJ. Transcranial magnetic stimulation for the treatment of migraine aura? Cephalalgia 29(Suppl. 1), 22 (2009).

$\checkmark 2$ Lipton RB, Dodick DW, Silberstein SD et al. Single-pulse transcranial magnetic stimulation for acute treatment of migraine with aura: a randomised, double-blind, parallel-group, sham-controlled trial. Lancet Neurol. 9(4), 373-380 (2010).

3 Weatherall MW, Bhola R, Giffin N, Goadsby PJ. Post market pilot programme with single pulse transcranial magnetic stimulation (sTMS) for acute treatment of migraine: SpringTMS $\left({ }^{\mathrm{TM}}\right)$ use in migraine. Presented at: 3rd EHMTIC, London, UK, 20-23 September 2012.

4 Vigano A, Bogdanov V, Schoenen J. A multidisciplinary approach to the functional abnormalities of the migrainous brain and non-invasive interventions to treat them. Presented at: $3 r d$ EHMTIC, London, UK, 20-23 September 2012.

-5 Magis D, Schoenen J. Advances and challenges in neurostimulation for headaches. Lancet Neurol. 11(8), 708-719 (2012).

6 Vigano A, Magis D, Sava SL et al. Anodal transcranial direct current stimulation of the visual cortex for migraine prevention: a proof-of-concept study. Presented at: $3 r d$ EHMTIC, London, UK, 20-23 September 2012.

7 Magis D, Gerard P, Schoenen J. Transcutaneous vagus nerve stimulation (tVNS) for headache prophylaxis: initial experience.
Presented at: 3rd EHMTIC, London, UK, 20-23 September 2012.

8 Nesbitt AD, Marin JC, Goadsby PJ. Treatment of hemicrania continua by non-invasive vagus nerve stimulation in 2 patients previously treated with occipital nerve stimulation. Presented at:

3rd EHMTIC, London, UK, 20-23

September 2012.

9 Schoenen J, Vandersmissen B, Jeangette S et al. Prevention of Migraine by supraorbital transcutaneous neurostimulation using the Cefaly ${ }^{\circledR}$ device (PREMICE): a multi-centre, randomized, sham-controlled trial. Presented at: $3 r d$ EHMTIC, London, UK, 20-23 September 2012 (In Press).

10 Schoenen J, May A, Jensen R et al. Pathway ch-1 study: sphenopalatine ganglion (spg) stimulation for acute treatment of chronic cluster headache $(\mathrm{CCH})$. Presented at: $3 r d$ EHMTIC, London, UK, 20-23 September 2012.

11 Lainez M, Jensen R, May A et al. Pathway $\mathrm{CH}-1$ study: sphenopalatine ganglion (SPG) stimulation for acute treatment of chronic cluster headache $(\mathrm{CCH})$ - initial experience (S36.002). Neurology 78 (Meeting Abstracts 1), S36.002 (2012).

$\checkmark 12$ Magis D, Allena M, Bolla M, De Pasqua V, Remacle J-M, Schoenen J. Occipital nerve stimulation for drug-resistant chronic cluster headache: a prospective pilot study. Lancet Neurol. 6(4), 314-321 (2007).

Burns B, Watkins L, Goadsby PJ. Treatment of medically intractable cluster headache by occipital nerve stimulation: long-term follow-up of eight patients. Lancet 369 (9567), 1099-1106 (2007).

14 Burns B, Watkins L, Goadsby PJ. Treatment of intractable chronic cluster headache by occipital nerve stimulation in 14 patients. Neurology 72(4), 341-345 (2009).

$\checkmark 15$ Magis D, Gerardy PY, Remacle JM, Schoenen J. Sustained effectiveness of occipital nerve stimulation in drug-resistant chronic cluster headache. Headache 51(8), 1191-1201 (2011).

6 Fontaine D, Christophe Sol J, Raoul S et al. Treatment of refractory chronic cluster headache by chronic occipital nerve stimulation. Cephalalgia 31(10), 1101-1105 (2011).

17 Saper JR, Dodick DW, Silberstein SD, McCarville S, Sun M, Goadsby PJ; ONSTIM Investigators. Occipital nerve stimulation for the treatment of intractable chronic migraine headache: ONSTIM feasibility study. Cephalalgia 31(3), 271-285 (2011).

- 18 Popeney CA, Aló KM. Peripheral neurostimulation for the treatment of chronic, disabling transformed migraine. Headache 43(4), 369-375 (2003).

- 19 Schwedt TJ, Dodick DW, Hentz J, Trentman TL, Zimmerman RS. Occipital nerve stimulation for chronic headache long-term safety and efficacy. Cephalalgia 27(2), 153-157 (2007).

-20 Magis D, Bruno MA, Fumal A et al. Central modulation in cluster headache patients treated with occipital nerve stimulation: an FDG-PET study. BMC Neurol. 11, 25 (2011). 